\title{
Sozialpolitik als Problemarbeit an Menschen und an Institutionen
}

\author{
Hannu Turba
}

Eingegangen: 1. August 2020 / Angenommen: 24. September 2020 / Online publiziert: 28. Oktober 2020 (C) Der/die Autor(en) 2020

Zusammenfassung In der Soziologie sozialer Probleme ist Sozialpolitik seit jeher ein wichtiger Aspekt, wird aber nur selten explizit in den Mittelpunkt gestellt. Der Beitrag verdeutlicht die Relevanz von Sozialpolitik sowie ihrer organisationalen und professionellen Bezugspunkte (Wohlfahrtspflege, soziale Dienstleistungsberufe) im Kontext der Soziologie sozialer Probleme und nimmt eine Einordnung im Rahmen unterschiedlicher institutioneller Logiken vor. Mit Blick auf das Wechselspiel von Definitions- und Bearbeitungsprozessen ,von unten“ und deren Strukturierung ,von oben' werden mikrofundierte Perspektiven des soziologischen Neo-Institutionalismus und damit verwandte Ansätze als Bezugspunkte der Soziologie sozialer Probleme konturiert. Sozialpolitik wird dabei gleichermaßen als Arbeit an Menschen sowie als Arbeit an Institutionen begriffen. Es folgen kompakte Betrachtungen ausgewählter Politikfelder - Kinderschutz und Arbeitsverwaltung - aus Sicht der entfalteten Systematik sowie ein Fazit mit Perspektiven für die Forschung und Theorieentwicklung an der Schnittstelle zwischen der Soziologie sozialer Probleme und der Soziologie der Sozialpolitik.

H. Turba $(\bowtie)$

Institut für Sozialwesen, Abt. Sozialpolitik, Recht und Soziologie, Universität Kassel,

Arnold-Bode-Str. 10, 34127 Kassel, Deutschland

E-Mail: h.turba@uni-kassel.de 


\section{Social Policy, Social Problems Work and Institutional Work}

Abstract Social policy has always been highly relevant to the sociology of social problems, but it is rarely dealt with explicitly in the respective literature. The article illustrates how social policy, social welfare provision and human service professions matter when studying social problems from a sociological perspective. Drawing on institutional theory and its microfoundations, social policy making is considered through the lens of different institutional logics, understanding it as both 'social problems work' and 'institutional work'. The utility of this approach is demonstrated by a condensed analysis of two policy areas (child protection and employment administration). Finally, the article presents suggestions for future research and theory development at the intersection of the sociology of social problems and the sociology of social policy.

\section{Soziale Probleme und Sozialpolitik: Eine vernachlässigte Perspektive?}

Eine naheliegende Herangehensweise an einen thematisch zugeschnittenen Artikel in einem Jubiläumsheft ist es, zunächst einmal das zu würdigende Journal selbst zu konsultieren. Die Zeitschrift „Soziale Probleme“, deren Jubiläum mit der vorliegenden Ausgabe begangen wird, hat in ihrer 30-jährigen Geschichte rund 300 Beiträge versammelt. Eine Schlagwortsuche nach den Begriffen „Sozialpolitik“ und „Wohlfahrt“ in den Titeln dieser Beiträge liefert überraschenderweise lediglich drei Ergebnisse. Der erste Treffer - „Sozialstrukturelle Modernisierung: Stabilisierung oder Destruierung des Wohlfahrtsstaates“ (Krüger 1994) - greift mit seinem Bezug auf „Wohlfahrtskulturen“ ein nach wie vor aktuelles Thema der Sozialpolitikforschung auf (vgl. Pfau-Effinger 2019). Susanne Karstedts Artikel zum Thema „Recht und soziales Kapital im Wohlfahrtsstaat“ (1997) untersucht den mit Blick auf soziale Probleme besonders interessanten Nexus zwischen den Systemen sozialer Sicherung und strafrechtlicher Sozialkontrolle. Der dritte Beitrag zu „Personenzentrierung als sozialpolitische Programmformel im Zeichen der Inklusion“ (Schäfers 2014) dekonstruiert und hinterfragt schließlich in plausibler Weise einen politisch nutzbaren „Modebegriff“ und verweist auf damit verbundene Widersprüchlichkeiten. All dies sind gleichermaßen lesenswerte wie immer noch aktuelle Beiträge im Kontext Sozialpolitik. Was die reine Quantität betrifft, mag diese Ausbeute in der Gesamtschau des publizierenden Organs allerdings gering erscheinen - gerade wenn man bedenkt, welch hoher Stellenwert sozialpolitischen Hintergründen im Zusammenhang mit sozialen Problemen in der Vergangenheit teilweise eingeräumt worden ist. So schrieb Günter Albrecht im allerersten in „Soziale Probleme“ erschienenen Beitrag:

[F]ür die gesellschaftliche Bearbeitung sozialer Probleme sind insbesondere die Phasen der Formulierung und der Implementation einer Politik zur Lösung bzw. Umdefinition des Problems von zentraler Bedeutung, nicht nur weil sich das auf die Lage der Betroffenen auswirkt, sondern auch weil gerade diese Reaktionen in Politik und Verwaltung als Auslöser, Verursacher und Definitionsinstanz neuer sozialer Probleme bzw. alter sozialer Probleme im neuen 
Gewand von immer größerer Bedeutung werden, eine Begleiterscheinung des

Wohlfahrtsstaates, die wir für unsere Thematik nicht wichtig genug nehmen können (Albrecht 1990, S. 12).

Wenngleich Sozialpolitik also eher selten explizit und prominent erwähnt worden ist, liegt vor diesem Hintergrund die Vermutung nahe, dass das Thema in vielen Diskussionen trotzdem immer irgendwie dabei war oder zumindest implizit mitgedacht wurde.

Allerdings ist auch das allgemeinere Schlagwort „Politik“ mit weiteren 13 Treffern nicht gerade häufig vertreten. Davon entfallen allein sechs auf ein Schwerpunktheft zu „Drogenkonsum, Drogenproblemen und Drogenpolitik in Europa“ (Hrsg. Duprez und Groenemeyer 2009). Die zentrale These in diesem Zusammenhang lautet: Nicht das Problem bestimmt die Politik, sondern die Politik das Problem (vgl. ebd., S. 7). Dies ist auch mit Blick auf aktuelle Debatten in der Soziologie der Sozialpolitik, die die fragliche Politik nicht nur als „Problemlöser“, sondern auch als „Problemverursacher“" sehen (vgl. Lessenich 2019), wiederum ein hochrelevanter Befund.

Die übrigen sieben Beiträge thematisieren unter anderem „Die Medien als Organ demokratischer Kontrolle staatlicher Politik am Beispiel der Gesetzgebungsverfahren zum Demonstrationsstrafrecht“ (Floerecke und Mansel 1990), „Gesellschaftliche Entwicklung und Sanktionseinstellungen - Anmerkungen zur deutschen kriminalpolitischen Diskussion“ (Sack 2006), „Die Politische Ökonomie der Sicherheit“ (Klimke 2013) oder „Ein goldenes Zeitalter des Jugendstrafrechts? Politische Debatten über Jugendkriminalität in den 1970er und 1980er Jahren in der Bundesrepublik“ (Lampe 2016). Hier dominieren erkennbar Bezüge zum Politikfeld Kriminalität und Sicherheit. Ein weiterer Aufsatz zu „Politik gegen sozialräumlich konzentrierte Benachteiligung“ (Ratzka 2005) bezieht klar politisch Stellung, wenn geschlossen wird, „dass Quartiersmanagement und Bürgerbeteiligung angesichts des Umbaus zum aktivierenden Sozialstaat nicht als Ersatz für eine umfassende Sozialpolitik gesehen werden dürfen, wenn Probleme benachteiligter Quartiere dauerhaft gelöst werden sollen“ (ebd., S. 54).

Die mit Blick auf die (Theorie-)Entwicklung der Disziplin grundlegendsten Beiträge stammen schließlich von Axel Groenemeyer, dessen Gedenken das vorliegende Heft gewidmet ist. In seinem Aufsatz „Gesellschaftspolitische Relevanz und soziologische Reputation. Eine kleine Geschichte über 30 Jahre Soziologie sozialer Probleme in Deutschland“ (Groenemeyer 2006) thematisierte er seinerzeit ebenfalls ein Jubiläum (nämlich das der Sektion ,Soziale Probleme und Soziale Kontrolle“ in der Deutschen Gesellschaft für Soziologie) und bezog sich dabei sowohl auf die gesellschaftliche und politische Bedeutung des fraglichen Forschungsprogramms als auch auf aus seiner Sicht notwendige Neujustierungen bestimmter Fragestellungen und Konzepte. Er beschreibt dabei die Wurzeln der Soziologie sozialer Probleme als ,,anwendungsorientierte Perspektive der politischen Gesellschaftsreform“ (ebd., S. 10), also als eine Disziplin, die gewissermaßen selbst aktiv Politik betreiben wollte. Später erfolgte im Zuge einer Hinwendung zum Konstruktivismus eine tendenzielle Abkehr von dieser nunmehr als ,technokratisch verstandenen Praxisorientierung“ (ebd., S. 12). Bei allem, was damit analytisch gewonnen wurde, scheint Groenemeyer hier 
den Umstand, dass ,der Bezug zur Politik und zu Fragen der gesellschaftspolitischen Relevanz der Soziologie sozialer Probleme weitgehend verloren gegangen ist" (ebd., S. 14), unterm Strich eher zu bedauern.

Schließlich wäre da noch Groenemeyers äußerst inspirierender Beitrag ,Von der Sünde zum Risiko? - Bilder abweichenden Verhaltens und die Politik sozialer Probleme am Ende des Rehabilitationsideals“ (2001), der damals zentrale theoretische Überlegungen neu kombinierte und damit einen weiteren wichtigen Bezugspunkt zwischen der wissenschaftlichen Debatte über soziale Probleme und Sozialpolitik markiert. Konkret adressiert Groenemeyer hier zwei aus seiner Sicht systematisch vernachlässigte Aspekte in der Soziologie sozialer Probleme: Gesellschaftlichen Wandel und Politik (vgl. dazu Groenemeyer 2001, S. 147 f.). Den Grund für diese Vernachlässigung sucht er wiederum im Kontext der interaktionistisch-konstruktivistischen Wende der Disziplin, in deren Fahrwasser eine Hinwendung zu Strukturen und Entwicklungen auf der gesellschaftlichen Makroebene tendenziell mit dem Risiko des „Rückfalls“ in einen überkommenen „Objektivismus“ assoziiert wurde (ebd., S. 147). Stattdessen dominierte die mikroanalytische Beschäftigung mit Definitionen, Interpretationen und Rhetoriken verschiedener Akteure - dass diese unter Umständen Teil staatlicher Politik werden (können), erschien dabei eher als Nebenprodukt bzw. logische Konsequenz des eigentlich interessierenden vorgelagerten Problematisierungsprozesses (vgl. ebd., S. 148). Mit Blick auf den bereits erwähnten Befund, dass Politik soziale Probleme selbstverständlich (mit) konstituiert, wenn nicht sogar verursacht, schien dies Groenemeyer schon damals nicht konsequent zu Ende gedacht (ebd.). Er fährt fort:

Der Zusammenhang zwischen Politik und sozialen Problemen ist durchaus komplex, klar dürfte allerdings sein, dass sich soziale Probleme und Politik immer in einem Wechselverhältnis zwischen gesellschaftlichen und politischadministrativen Akteuren konstituieren. So basiert jede Politik auf bestimmten Auffassungen, Interpretationen oder Definitionen sozialer Probleme, die ihr Legitimation und Sinn verleihen. Damit können dann die verschiedenen politischen oder staatlichen Interventionen und die mit ihnen verbundenen Diskurse als zentrale Indikatoren für die jeweils offiziell anerkennte Konstruktion eines sozialen Problems angesehen werden. In diesem Sinne stellt die jeweilige Form der Politik sozialer Probleme die erfolgreiche Institutionalisierung eines bestimmten Diskurses über ein soziales Problem dar. (ebd.)

Während hier bereits der Begriff der Institutionalisierung auftaucht, sprach Groenemeyer mit Blick auf die Systematik unterschiedlicher Logiken der Problembearbeitung, die seinerzeit im Zentrum seines Beitrags standen, noch von „Repräsentationen abweichenden Verhaltens und Formen sozialer Kontrolle“ (ebd., S. 151). In der späteren Theorieentwicklung hat er diesen Begriff systematisch wieder aufgenommen und die fraglichen Logiken als „Institutionen der Normativität“ bezeichnet, womit er auf die „Verfestigung von Handlungsroutinen in Form eines geteilten nichthinterfragten Orientierungs- und Regelwissens“ und ,kulturelle Selbstverständlichkeiten“ verweist (Groenemeyer 2008, S. 86). Wenn es im vorliegenden Artikel um Sozialpolitik und deren organisationale und professionelle Bezugspunkte (Wohlfahrtspflege, soziale Dienstleistungsberufe etc.) im Kontext der Soziologie sozialer 
Probleme geht, wird zunächst dieser Begriffstradition gefolgt, also eine Einordnung von Sozialpolitik im Rahmen unterschiedlicher Institutionen der Normativität vorgenommen (Abschn. 2). Mit Blick auf das oben angedeutete Wechselspiel zwischen Definitions- und Bearbeitungsprozessen ,von unten“ und deren Strukturierung, von oben' werden im nächsten Schritt mikrofundierte Perspektiven des soziologischen Neo-Institutionalismus - sowie verwandte Ansätze - als Bezugspunkte der Soziologie sozialer Probleme konturiert: Sozialpolitik wird dabei gleichermaßen als Arbeit an Menschen sowie als Arbeit an Institutionen begriffen (Abschn. 3). Es folgen kompakte Betrachtungen ausgewählter Politikfelder - Kinderschutz und Arbeitsverwaltung - aus Sicht der entfalteten Systematik (Abschn. 4) sowie ein Fazit mit Perspektiven für die Forschung und Theorieentwicklung an der Schnittstelle zwischen der Soziologie sozialer Probleme und der Soziologie der Sozialpolitik.

\section{Sozialpolitik als Steuerungsmodus und Institution der Normativität}

Die zeitgenössische Soziologie sozialer Probleme, auf die Groenemeyer in den genannten Beiträgen Bezug nimmt, ist stark vom Konstruktivismus und symbolischen Interaktionismus sowie - in Bezug auf abweichendes Verhalten - vom Etikettierungsansatz geprägt. Und in der Tat wäre jede soziologische Perspektive auf soziale Probleme, die nicht deren soziale Konstruiertheit mit in den Blick nimmt, zu kurz gedacht. Zugleich gilt es aber auch, die Bedingungen von Zuschreibungs- und Aushandlungsprozessen zu untersuchen, gerade weil letztere in modernen Gesellschaften nicht völlig beliebig - quasi ,strukturenthoben“ (vgl. Peters und CremerSchäfer 1975, S. 78) - verlaufen. Hier sind Kräfte am Werk, für die die Soziologie den Begriff der Institutionen geprägt hat: Diese entstehen (wie alles Soziale) ursprünglich aus Konstruktions- und Aushandlungsprozessen, verselbstständigen sich aber derart, dass sie kaum noch hinterfragt werden und - einmal in der Welt - ein relativ stabiles Steuerungspotenzial mit Blick auf die gesellschaftliche Konstruktion von Wirklichkeit entfalten (vgl. Berger und Luckmann 1969). Dabei kann es sich sowohl um ,ungeschriebene Gesetze “ im Sinne kultureller Selbstverständlichkeiten als auch um rechtlich fixierte Normen und Regeln handeln.

Spätestens in letzterem Zusammenhang kommt ,die Politik - als Steuerungsinstanz - ins Spiel, die solche allgemein verbindlichen Regeln herbeiführt. Das politische System tritt hier gewissermaßen als ,großer Problemlöser“ (Groenemeyer et al. 2012, S. 118) auf den Plan, welcher bestimmte gesellschaftliche Forderungen und wahrgenommene Lösungsbedarfe aufnimmt und in Entscheidungen transformiert. Entlang solcher Input-Output-Modelle und ,,policy cycles“ beschäftigen sich politikwissenschaftliche Ansätze etwa mit Prozessen des Agenda Settings, der Entstehung politischer Issues, der Implementation und den Effekten bestimmter Politiken. ${ }^{1}$

\footnotetext{
1 Solche Perspektiven bleiben im vorliegenden Beitrag weitgehend ausgespart (vgl. dazu ausführlich Groenemeyer et al. 2012). Mit Blick auf das Weitere ist aber festzuhalten, dass die politische Thematisierung sozialer Probleme in hohem Maße vom jeweiligen institutionellen Kontext abhängig ist und den genannten Karriere- bzw. Phasenmodellen demnach eher heuristische Funktionen zukommen (Groenemeyer 2010, S. $20 \mathrm{f}$.).
} 
Versteht man Politik in diesem Sinne, so geht es dabei immer auch um (Konflikte um) die Durchsetzung von Macht, was darauf verweist, dass gerade in dieser gesellschaftlichen Sphäre Potenziale für Komplexität und institutionellen Wandel besonders deutlich zu Tage treten: Bezogen auf unseren Gegenstand bedeutet dies, dass unterschiedliche Vorstellungen darüber in der Welt sind, was ein soziales Problem ,ist", dass diese Vorstellungen prinzipiell strittig sind und sich deshalb im Laufe der Zeit verändern können.

Darüber hinaus lässt sich Sozialpolitik im gegebenen Zusammenhang aber nicht nur als bestimmter Steuerungsmodus, sondern auch als eigenständige „KontrollLogik“ (Turba 2018, S. $20 \mathrm{ff}$.) innerhalb einer Gesamtsystematik sozialer Probleme und deren Bearbeitung, also - wiederum Groenemeyer $(2001,2008)$ folgend - als eine von verschiedenen „Institutionen der Normativität“ verstehen. Letztere lassen sich einerseits chronologisch historischen Epochen ihres Entstehens bzw. ihrer Hegemonie zuordnen und sind andererseits in der fortgeschrittenen Moderne gleichzeitig in der Welt: Neben religiös geprägten Vorstellungen von Sünde und Läuterung, dem rechtsstaatlich verankerten Komplex von Kriminalität und Strafe, Therapie bzw. Hilfe durch Professionen (Medizin und Soziale Arbeit) angesichts diagnostizierter Krankheiten oder Sozialisationsdefizite sowie auf Prävention und Kompensation ausgerichtetem Management von Risiken wird auch Sozialpolitik dieser Systematik zufolge im Rahmen eines eigenständigen institutionellen Zusammenhangs verstanden - konkret jenem, der sich mit dem Problem der „Deprivation/Armut" befasst (Groenemeyer 2008, S. 87 ff.). Zusammen mit den genannten ,professionellen“ Diskursen (Behandlung von Krankheit und Hilfe bei Defiziten) wird die Logik der Sozialpolitik unter dem gemeinsamen Dach des „Rehabilitationsideals" verortet, welches die Ursachen für Abweichung jenseits individueller Verantwortlichkeit sucht und gleichzeitig die Möglichkeit positiver Veränderungen von Personen in Betracht zieht (ebd.). Für eine idealtypische Vergewisserung über das in Gegenwartsgesellschaften ,,begrenzte“ Repertoire an Problemdeutungen und damit verbundenen Kontrollpraktiken (Groenemeyer 2008, S. 86) erscheint diese Systematisierung nach wie vor schlüssig und vielversprechend.

Geht man allerdings ins Detail, so weist die idealtypische Charakterisierung von Sozialpolitik - innerhalb der genannten Gesamtsystematik - mehr Bruchstellen auf als andere Institutionen der Normativität. Dies hängt einerseits mit dem komplexen Institutionengefüge zeitgenössischer Wohlfahrtsstaaten zusammen, welches ein breites Spektrum an - rechtlichen, ökonomischen, ökologischen und pädagogischen - Interventionsformen umfasst (vgl. Kaufmann 2002, 2012). So beinhaltet Sozialpolitik z. B. nicht nur ökonomische Transferleistungen, sondern auch soziale Dienstleistungen und bedingt somit in hohem Maße die (Infra-)Strukturen und Möglichkeitsspektren, innerhalb derer sich die Soziale Arbeit bewegt (vgl. Kaufmann 2012, S. $1301 \mathrm{ff}$.). Versteht man Gesundheitspolitik als Teil von Sozialpolitik, so gilt dies auch für die Pflege- und Medizinberufe. Die beteiligten Professionen entfalten auf Basis ihres spezifischen Wissens und Berufsethos eine Eigenlogik, welche sich auch jenseits des individuellen Falls - auf die Diagnose von und den Umgang mit sozialen Problemen auswirkt. Insofern macht es durchaus Sinn, im o.g. Spektrum des Rehabilitationsideals mehrere Logiken zu unterscheiden. Davon abgesehen spricht aber vieles dafür, Sozialpolitik nicht als gleichwertige Kategorie neben den 
anderen, sondern eher als komplementären Bestandteil bzw. Oberbegriff im Kontext verschiedener anderer Logiken zu begreifen - gerade weil sie niemals losgelöst von dem betrachtet werden kann, was vor Ort aus politischen Programmen gemacht wird (dazu unten mehr).

Auch mit Blick auf die dahinterliegenden Leitideen ist Sozialpolitik uneindeutiger als andere Kontroll-Logiken - so kann die normative Zielrichtung von Politik vorrangig an Idealen von Sicherheit, Gleichheit oder auch Freiheit ausgerichtet sein (Bäcker et al. 2010, S. 53 ff.; vgl. auch Kaufmann 1973, S. 37; sowie Nullmeier 2019, S. 67 ff.). Damit verbundene Gerechtigkeitsvorstellungen sind politischen Aushandlungs- und historischen Wandlungsprozessen unterworfen, existieren aber in Gegenwartsgesellschaften gleichzeitig bzw. nebeneinander, weshalb eine grundsätzliche „Widersprüchlichkeit“ von Sozialpolitik zu konstatieren ist (Bäcker et al. 2010, S. 56; vgl. Lessenich 2019, S. 897 f.). In diesem Sinne sind in manchen Politikfeldern auch Schnittstellen mit anderen Kontroll-Logiken festzustellen, die der oben entfalteten Systematik zufolge außerhalb des Rehabilitationsideals zu verorten sind - etwa wenn in der Arbeitsverwaltung mit „strafenden“ Sanktionen (Gurr 2018) oder in der Kinder- und Jugendhilfe mit neuen Formen des "Risikomanagements“ (Wolff 2017) operiert wird. ${ }^{2}$

All dies spricht für ein hohes Maß an institutioneller Komplexität mit Blick auf die Bearbeitung sozialer Probleme. Ein geeignetes Instrumentarium zur Analyse solcher Komplexität stellen neuere Ansätze des soziologischen Neo-Institutionalismus dar, welcher der - auch in der Soziologie sozialer Probleme verankerten - sozialkonstruktivistischen Tradition folgt, ursprünglich aber vor allem mit besonderer Deutungsmacht ausgestattete überindividuelle bzw. kollektive Instanzen wie Organisationen, Professionen oder auch die staatliche Politik in den Blick nimmt und dabei insbesondere die Komplexität und Dynamik von Institutionen fokussiert (vgl. Turba 2018, S. 16). Traditionell befassen sich solche Ansätze mit der Einbettung von Organisationen in übergeordnete gesellschaftliche Strukturen und institutionelle Umwelten sowie deren Wandel. Institutionen werden dabei aber nicht als objektiv gegeben, sondern als Ergebnis sozialer Konstruktionen, im o. g. Sinne also als „Sedimentierungen" von Deutungsschemata individueller bzw. organisierter Akteure, verstanden (vgl. dazu Drepper 2010, S. 136). Zugleich sind akteursseitige Zuschreibungs- und Verarbeitungsprozesse auf der Mikroebene ebenfalls Teil des fraglichen Theorieprogramms (vgl. Powell und Colyvas 2008; Hasse und Schmidt 2010) und haben in den letzten Jahren nochmals deutlich an Bedeutung gewonnen. Insofern wird gewissermaßen eine Brücke zwischen ,Konstruktivismus “ und ,Objektivismus “ geschlagen, die - nicht zuletzt angesichts der zuvor angedeuteten Bruchlinien - auch für die Soziologie sozialer Probleme aussichtsreich erscheint. Diese Perspektive wird im folgenden Abschnitt weiterverfolgt.

\footnotetext{
2 Diese Beispiele werden in Abschn. 4 des vorliegenden Beitrags vertieft betrachtet.
} 


\section{Sozialpolitik als Arbeit an Menschen und an Institutionen}

Wesentliche Parallelen zwischen der Soziologie sozialer Probleme und dem soziologischen Neo-Institutionalismus liegen im theoretischen Begriff der Arbeit begründet (vgl. dazu nachfolgend Schmidt und Hasse 2010, S. 57 ff.). Dabei lassen sich zwei Aspekte unterscheiden: Der Umgang mit sozialen Problemen kann einerseits (auf der Mikroebene) als Arbeit an Menschen sowie andererseits (makrosoziologisch) als Arbeit an Institutionen aufgefasst werden. Wie im Folgenden gezeigt wird, hat beides mehr oder weniger direkt mit Sozialpolitik zu tun.

Was die Makroebene betrifft, so lassen sich zunächst Ähnlichkeiten zwischen dem für die Soziologie sozialer Probleme prägenden Begriff der „claimsmaking activities" (Spector und Kitsuse 1977) und neo-institutionalistischen Vorstellungen von ,institutional entrepreneurship“ (DiMaggio 1988) ausmachen: Beide gehen von relativ autonomen, durchsetzungsstarken Akteuren aus, die - etwa als Mitglieder von Parteien und Verbänden oder im Rahmen öffentlich-medialer Diskurse - in der Lage sind, auf politische Thematisierungs- und Aushandlungsprozesse Einfluss zu nehmen. Hier liegt der Bezug zu Sozialpolitik also unmittelbar auf der Hand.

Nicht minder bedeutsam ist der Nexus von Sozialpolitik und der Arbeit an oder mit Menschen, wie sie z.B. in sozialen Dienstleistungsorganisationen stattfindet. Dahinter steht die Annahme, dass nicht alle Akteure, die soziale Probleme (mit) konstituieren zwingend in öffentlichen Diskursarenen besonders exponierte ,Aktivist/innen " sind: „For some, social problems are just another day at the office“ (Hilgartner und Bosk 1988, S. 57). Ausgehend von dieser Einsicht fokussierte die Forschung in den 90er Jahren verstärkt auf ,social problems work“, verstanden als die alltägliche Definitions- und Legitimationsarbeit in Organisationen, die vor Ort mit sozialer Hilfe und Kontrolle befasst sind (Miller und Holstein 1997).

Neo-institutionalistisch geprägte Ansätze zu solchen „human service organizations" nehmen eine ähnliche Sichtweise ein, indem sie deren Arbeit als „,moral work“ charakterisieren (Hasenfeld 2010a): Humandienstleistungen werden dabei als eine Arbeit am „Rohmaterial Mensch“ verstanden, die das Ziel verfolgt, Personen zu verändern (,,people changing“) bzw. ,weiterzuverarbeiten“ (,,people processing“). Da das ,Rohmaterial " von besonderer Beschaffenheit ist, setzt diese Arbeit unweigerlich moralische Werturteile voraus, die eng mit der Konstitution sozialer Probleme zusammenhängen. Wie Hasenfeld (2000) am Beispiel von Sozialbehörden im Umgang mit armen, alleinerziehenden Müttern eindrucksvoll zeigt, vollziehen sich Prozesse der Problemattribution auf unterschiedlichen Ebenen - neben der ,großen Politik ${ }^{\text {‘ }}$ sind es auch lokale ,communities', die Organisationen selbst und nicht zuletzt Mitarbeiter/innen an der Basis, die mit Blick auf die Kategorisierung ihrer Zielgruppen Maßstäbe setzen und im Rahmen dieses „Moralunternehmertums“ auch historischen Wandel mit bedingen (ders., S. 348).

Letztere These findet sich in zugespitzter Form auch in einflussreichen Arbeiten zu „street level bureaucracies“ (Lipsky 2010 [1980]): Im Gegensatz zu klassischen Bürokratievorstellungen wird bestimmten ,front-line workers“ im öffentlichen Dienst (z.B. Sozialarbeiter/innen, Polizist/innen, Lehrkräften, Beschäftigten in Rechtspflege und Gesundheitswesen) dabei ein ausgeprägter Ermessensspielraum zugeschrieben. Vor diesem Hintergrund wird argumentiert, dass sich die Entschei- 
dungen, Routinen und kreativen Umgangsformen mit alltäglichen Dilemmata dieser Akteure vor Ort kaum sinnvoll als Ergebnis, von oben' oktroyierter politischer Entscheidungen deuten lassen - vielmehr verkörpern sie im Ergebnis gewissermaßen selbst diese Politik. Sozialpolitik wird demnach nicht von Regierungen, Führungsgremien oder Behördenleitungen gemacht, sondern von professionellen Fachkräften und Sachbearbeiter/innen an der Basis (vgl. Lipsky 2010, S. xiii). Michael Lipsky hat 30 Jahre nach der Erstveröffentlichung selbst darauf verwiesen, dass diese plakativ anmutende These so häufig verkürzt wiedergegeben worden ist und bei der Betrachtung politischer Prozesse natürlich auch die Strukturen und Kontexte berücksichtigt werden müssen, in die die Arbeit von ,street level bureaucrats“ eingebettet ist (ders., S. $221 \mathrm{f}$.). Jedenfalls ist daraus eine Vielzahl von Forschungsarbeiten in unterschiedlichen Bereichen entstanden, die insgesamt für den Stellenwert von ,frontline work" in sozialpolitischen Zusammenhängen sensibilisieren (Nothdurfter und Hermans 2018). Wenn oben von einer Arbeit an Menschen und an Institutionen die Rede war, so wird vor diesem Hintergrund umso deutlicher, wie stark diese beiden Ebenen miteinander verwoben sind.

Solche Ansätze, die die Dualität von Prozessen auf der Mikro- und der Makroebene gezielt in den Blick nehmen, erweisen sich auch für die Soziologie sozialer Probleme als anschlussfähig. Axel Groenemeyer hat in seinem Forschungsprogramm zu "Mikroanalysen der Konstruktion sozialer Probleme in institutionellen Kontexten“ unter der Überschrift „Doing Social Problems“ bereits vieles davon ausbuchstabiert, wobei es ihm nicht zuletzt ein Anliegen war, die Einbettung von Problemarbeit in gesellschaftliche und politische Diskurse mitzudenken und eine gemeinsame konzeptionelle Grundlage für die integrierte Betrachtung unterschiedlicher Institutionen der Normativität zu schaffen (Groenemeyer 2010, S. 51 f.). Damit scheint er das Feld der Soziologie sozialer Probleme relativ breit abzustecken. Schmidt und Hasse (2010, S. $73 \mathrm{f}$.) fügen im gleichen Band an, dass das von ihnen skizzierte Forschungsprogramm - an der Schnittstelle zum Neo-Institutionalismus - nicht nur geeignet sei, die „üblichen Verdächtigen“ (wie z.B. Polizei oder Sozialarbeit) zu beforschen, sondern auch der Tatsache Rechnung zu tragen, dass „,z. B. die Notwendigkeit einer Inanspruchnahme von Einrichtungen der Sozialfürsorge nicht selten auch durch zeitlich vorgelagerte Problemarbeit in Schulen, Ausbildungsbetrieben und Wirtschaftsunternehmen (mit-)verursacht wird“. Wenn es um den Zusammenhang von Problemarbeit und Sozialpolitik geht, spricht - nicht zuletzt aufgrund der vielfältigen Anschlussstellen - in der Tat vieles für Konzepte, die gesellschaftliche und politische Kontexte möglichst umfangreich einbeziehen.

In diesem Zusammenhang sei einmal mehr auf die besagten Überschneidungen zwischen der Soziologie sozialer Probleme und neueren Varianten des Neo-Institutionalismus verwiesen, die ihren Fokus auf ,institutionelle Komplexität“ (Greenwood et al. 2011), das Nebeneinander unterschiedlicher ,institutioneller Logiken“ (Thornton et al. 2012; Friedland und Alford 1991) oder „,institutionelle Arbeit“ (Lawrence und Suddaby 2006) im Umgang mit dieser Komplexität legen. Gerade das Konzept von ,institutional work“ weist wiederum markante Ähnlichkeiten mit der o.g. Perspektive auf ,social problems work“ auf, indem es die Bedeutung der Arbeit individueller bzw. organisierter Akteure an der Herstellung, Aufrechterhaltung oder Zerstörung von Institutionen hervorhebt (dies., S. 215). Dabei geht es auch hier 
ausdrücklich nicht um durchsetzungsstarke „claimsmakers“ oder ,entrepreneurs“, sondern um eine Vielzahl von Personen, die mittels verschiedener Definitionsprozesse, rhetorischer Strategien und theoretischer (Neu-)Erfindungen quasi „,behind the scenes" institutionellen Wandel vorantreiben oder auch - z. B. auf dem Wege einer ,Verschleierung“ von Widersprüchen - für Stabilität sorgen (dies., S. $248 \mathrm{f}$.). ${ }^{3}$ Interessant ist diese Perspektive insbesondere in Feldern, die sich durch ein hohes $\mathrm{Maß}$ an institutioneller Komplexität, also eine Vielzahl an inkonsistenten Erwartungen und widersprüchlichen institutionellen Logiken auszeichnen. Wie im vorherigen Abschnitt anhand der vielfältigen Bezugspunkte und Reibungsflächen mit anderen Kontroll-Logiken und Institutionen der Normativität gezeigt wurde, ist dies bei sozialpolitischen Arrangements eher die Regel als die Ausnahme. Es spricht also vieles für eine Perspektive, die sowohl gesellschaftliche und makropolitische Dynamiken als auch die ,gelebte' Sozialpolitik in den Organisationen vor Ort - sowie deren Wechselverhältnis - in den Blick nimmt. Im Folgenden wird dies am Beispiel zweier konkreter Politikfelder näher ausbuchstabiert.

\section{Komplexität im Großen und im Kleinen: Ausgewählte Politikfelder}

Zunächst soll mit dem Politikfeld Kinderschutz ein Thema angesprochen werden, welches in vielen Ländern der Welt ein Paradebeispiel für institutionelle Komplexität darstellt. Die internationale Literatur beschreibt unterschiedliche Strömungen in der sozialpolitischen Ausrichtung dieses Feldes: eine Logik des „family service“, nach der mittels sozialer Dienstleistungsangebote versucht wird, Eltern zu einem gewaltfreien Umgang mit ihren Kindern zu befähigen; eine Orientierung an ,child protection“ im engeren Sinne, bei denen Eltern als potenzielle ,Täter/innen“ konstruiert werden, vor denen Kinder mittels staatlicher Kontrolle geschützt werden müssen; sowie ein Akzent auf ,child focus“, nach dem besonderer Wert darauf gelegt wird, das Kind als Akteur mit eigenen Bedürfnissen zur Kenntnis zu nehmen und entwicklungsfördernde Maßnahmen in diesem Sinne auszurichten (Gilbert et al. 2011, S. 251 ff.).

Folgt man der o.g. Systematik verschiedener Institutionen der Normativität (Groenemeyer 2008), so ist die erste Perspektive klar dem Rehabilitationsideal (konkret v. a. einer sozialarbeiterischen Logik), die zweite dagegen eher einer quasi-polizeilichen Logik der Eingriffsverwaltung zuzuordnen. Mit Blick auf internationale Vergleiche wird die Orientierung an ,family service“ in erster Linie mit den universalistischen Wohlfahrtsstaaten Skandinaviens in Verbindung gebracht, ,child protection“ dagegen v.a. mit den liberalen angelsächsischen Ländern - soweit ähnelt die Typisierung klassischen Einteilungen unterschiedlicher ,welfare regimes“ (vgl. Anttonen und Sipilä 1996). Mit dem Erstarken der ,child focus“-Perspektive sind die Verhältnisse allerdings vielerorts komplizierter geworden, was nicht nur analy-

\footnotetext{
3 Dabei wird im vorliegenden Beitrag einer Perspektive gefolgt, die (abweichend von der ursprünglichen Konzeption) unter ,,institutional work“ nicht nur absichtsvolles, wissentliches Handeln, sondern auch latente, habitualisierte Praktiken versteht (vgl. Hasse und Schmidt 2010; Schmidt und Hasse 2010, S. 66; Greenwood et al. 2011, S. 352; Turba 2018, S. 36).
} 
tische Fragen der Sortierung von Kinderschutzsystemen, sondern auch die Praxis betrifft. Marit Skivenes spricht diesbezüglich (mit Blick auf Norwegen, aber zugleich stellvertretend für viele andere Länder) von einer zunehmenden Vermischung von Perspektiven und daraus folgend ,,conflicting values and principles without indicating how to balance them in practice“" (Skivenes 2011, S. 172). ${ }^{4}$

So ist der Gedanke der Kindzentrierung (,child focus“) ursprünglich aus einem Menschenrechtsdiskurs entstanden, wird aber mittlerweile in verschiedenen Ländern auch mit Formen eines ,autoritären Risikomanagements“ (Wolff 2017) in Verbindung gebracht und sorgt deshalb verstärkt für öffentliche Kritik (Turba et al. 2019, S. 2). Wiederum in Anschluss an Groenemeyer lässt sich diese Entwicklung gewissermaßen im Sinne einer „Bifurkation“ beschreiben (ders. 2001, S. 167): Einerseits wird in Abkehr vom Rehabilitationsideal pragmatisch-nüchtern auf Risikoprävention gesetzt, wobei das Kind als zu schützendes Objekt (und zugleich als ,Sozialinvestition") im Mittelpunkt steht, andererseits aber ein Moraldiskurs entfacht, der (Gruppen von) Eltern, die bestimmten Normalitätsvorstellungen nicht entsprechen, als ,Täter/ innen'ins Abseits stellt (vgl. Mierendorff und Ostner 2014).

Hinzu kommt, dass die Steuerung des fraglichen Politikfelds traditionell zuvörderst den Bereich der Kinder- und Jugendhilfe betrifft, spätestens seit dem Bundeskinderschutzgesetz von 2012 aber zunehmend auch andere Organisationen und Berufsgruppen in die Praxis des Kinderschutzes einbezogen werden sollen. Darin liegt ein weiteres gewichtiges Moment von Komplexität (vgl. etwa zur Rolle der Polizei Turba 2018; zum Gesundheitswesen Sucherdt 2020). Im Feld dominante Rationalitäten vervielfältigen sich abermals und sorgen verstärkt für Reibungsflächen, denen die unterschiedlichen Professionen und Organisationen im Rahmen von ,institutional work" begegnen (müssen). Darüber hinaus treten sie durchaus auch im klassischen Sinne als „claimsmakers“ bzw. ,institutional entrepreneurs“ in Erscheinung, indem sie im Kontext ihrer jeweils eigenen Berufslogik bestimmte Moralvorstellungen und Problemkonstruktionen propagieren. ${ }^{5}$

Auch jenseits von Professionsgrenzen ist im Feld des organisierten Kinderschutzes schließlich mit vielseitigen politischen Dynamiken und Eigensinnigkeiten auf der Mikroebene zu rechnen - einschließlich Formen des Widerstands, die politische Vorgaben faktisch unterlaufen können (Bode und Turba 2014). Zudem ist die Varianz lokaler (Infra-)Strukturen erheblich, so dass Kinderschutz auf „street level“ so oder so ausgestaltet werden kann (ebd.). Es zeigt sich also ein hohes Maß an Ambiguität und Dynamik sowohl auf der Makro- als auch auf der Mikroebene, welches unterkomplex gefasst wäre, wenn lediglich sozialpolitische Programme betrachtet würden.

Ähnliches zeigt sich im Politikfeld der Arbeits(losen)verwaltung, welches nachfolgend als zweites Anschauungsbeispiel dient. Auch hier ist eine spezifische $M i$ schung unterschiedlicher institutioneller Referenzen im Zuge eines markanten Wan-

\footnotetext{
4 Ähnliche Ratlosigkeit zeigt sich dort, wo (wie in Deutschland) verstärkt klassische wohlfahrtsstaatliche mit managerialistischen Logiken zusammenprallen - oft bleibt dann nur ziellose Improvisation ohne Aussicht auf eine Auflösung der strukturell verankerten Konflikte (Bode und Turba 2020).

5 Gerade die Logik der Risikoprävention erweist sich dabei als vielseitig anschlussfähig und scheint deshalb zu Legitimationszwecken besonders ,geeignet“ (Turba 2018, S. 373).
} 
dels in Richtung ,aktivierender' Sozialpolitik in den letzten Jahrzehnten augenfällig. So gibt es Anhaltspunkte für ein (Wieder-)Erstarken ,,punitiver Sozialstaatsfunktionen“ und ,repressiver Instrumente“, die traditionell eher der Kriminalpolitik zuzuordnen wären, sich aber gleichzeitig offiziell als „Inklusionsmechanismus“ für mündige „Kund/innen“ verstehen (vgl. Dahme und Wohlfahrt 2011, S. 207 ff.). Sozialpolitik verbindet also Elemente von Hilfe und Strafe mit Vorstellungen von Sozialinvestition, was wiederum für eine Art Bifurkation (s. oben) sowie ein hohes Maß an institutioneller Komplexität spricht. Deshalb plädiert z. B. Dollinger (2007) dafür, Sozial- und Kriminalpolitik gar nicht mehr als separate Logiken, sondern gewissermaßen durch die gleiche analytische Brille als lebenslaufkonstituierende Institutionen zu betrachten, deren entscheidender Bestandteil Negativsanktionierungen sind. Auf ähnliche Parallelen zwischen strafrechtlichen und wohlfahrtsstaatlichen Steuerungsinstrumenten und Diskursen verweisen international z.B. Garland (2001) und Wacquant (2013).

Vielleicht deutlichster Ausdruck dieser Entwicklung hin zu einem „bestrafenden Management sozialer Ungleichheit und sozialer Probleme“" sind in Deutschland die negativen Sanktionen für junge Arbeitslose nach dem SGB II (Gurr 2018, S. 258). Die Forschung zu diesem Thema zeigt, dass sich diese Maßnahmen im Sinne eines „Integrationsversprechens“ für die Betroffenen nur bedingt eignen (ders., S. 252) und die ,widersprüchlichen Signale“, die damit einhergehen, von letzteren einseitig als Abwertung erfahren werden, was verschärfte Exklusionsprozesse nach sich ziehen kann (Zahradnik 2018, S. 287 ff.).

In diesem Zusammenhang ist einmal mehr zu betonen, dass die Art und Weise, wie Sozialpolitik auf „street level“ implementiert wird und welche Folgen dies für die betroffenen ,Kund/innen" hat, hochgradig von Kontexten und Dynamiken auf der Mikroebene abhängig ist (vgl. Wiedemeyer et al. 2018, S. 335; Kaufmann 2012, S. 1297f.). Darauf verweisen schon klassische Untersuchungen zur moralischen Kategorisierung von Adressat/innen in ,deserving“ und „,undeserving“ (Hasenfeld 2000). Dabei ist der lokale „Moralhaushalt“ offensichtlich nur lose mit politischen Programmen auf der Makroebene gekoppelt und stark von der interaktiven Beziehungskonstellation vor Ort geprägt (vgl. Hasenfeld 2010b, S. 410). So scheinen z. B. ein niedriger Bildungsstand und eine damit verbundene ,habituelle Distanz“ zwischen Adressat/innen und Fachkräften - unabhängig von der generellen Motivation und Mitwirkungsbereitschaft der Betroffenen - die Sanktionswahrscheinlichkeit zu erhöhen (Zahradnik et al. 2016), während umgekehrt diejenigen, die sich erfolgreich als ,hilfsbedürftig' zu inszenieren wissen (Böhringer et al. 2012, S. 241) oder die ,Sprache der Bürokratie“ beherrschen, privilegiert behandelt werden (LudwigMayerhofer et al. 2009, S. 113 ff.).

Teilweise mag es sich dabei um individuelle moralische Werturteile, teilweise aber auch um eingeschliffene Routinen handeln, die aus der lokalen Organisationskultur erwachsen (vgl. Altreiter und Leibetseder 2015). Denn schließlich erlaubt das Dispositiv der „Aktivierung“ individuelle Schuldzuweisungen an diejenigen, die nicht in das vorgesehene Schema hineinpassen, und eröffnet so Optionen einer moralischen „Entlastung“ für die zuständigen Organisationen (vgl. Ludwig-Mayerhofer et al. 2009). Gleichzeitig trägt es dazu bei, „den Fall bearbeitbar zu halten“ (vgl. Böhringer et al. 2012) und ermöglicht so gewissermaßen ein bürokratisches 
„business as usual“. Wer als unterstützungswürdig bzw. bestrafungsbedürftig definiert oder schlicht als ,hoffnungsloser Fall ' etikettiert wird, scheint jedenfalls von zahlreichen Faktoren abzuhängen, die mit sozialpolitischen Programmen an sich nur sehr eingeschränkt zu tun haben. Schließlich darf nicht vergessen werden, dass die relative Gestaltungsmacht der „Akteure des Sozialen“ (Bode 2013, S. 47f.) immer auch der Verwirklichung von Emanzipationszielen dienen kann - etwa wenn vor Ort Diskussionen in Richtung einer Beratungspraxis angestoßen werden, die die soziale Unterstützung von Betroffenen stärker betont (vgl. Wiedemeyer et al. 2018, S. 335 f.). „Institutional work“ als Antwort auf vorgefundene Komplexität kann also unterschiedliche Folgen haben und unter Umständen auch auf die Makroebene zurückwirken.

Das Anschauungsbeispiel zeigt wiederum, dass es für die wissenschaftliche Analyse von Sozialpolitik und sozialen Problemen aussichtsreich ist, beide Ebenen den gesetzlich-institutionellen Background, aber auch die versteckten Dynamiken auf ,street level“ - gemeinsam in den Blick zu nehmen.

\section{Fazit}

Ausgangspunkt des vorliegenden Artikels war die Beobachtung, dass dem Begriff der Sozialpolitik - zumindest gemessen an den Beitragstiteln in ,Soziale Probleme“vermeintlich keine besondere Prominenz in der (deutschen) Soziologie sozialer Probleme zukommt (vgl. dazu Groenemeyer et al. 2012, S. 117). Dabei dürfte unstrittig sein, dass das Auftauchen von Titelstichworten nur unvollständig über die Relevanz bestimmter Begriffe oder Konzepte in wissenschaftlichen Diskursen Auskunft gibt und solche Begriffskonjunkturen zudem von zahlreichen Faktoren abhängen. Eine Hypothese wäre, dass der Prozess der ,,Verwissenschaftlichung“ bei einer partiellen Abkehr vom „Praxisbezug“ der fraglichen Disziplin zur Geburtsstunde der Zeitschrift „Soziale Probleme“ längst abgeschlossen und zugleich die Hinwendung zu einer mikroanalytisch-konstruktivistischen Perspektive auf soziale Probleme bereits weit vorangeschritten war (vgl. Groenemeyer 2006, S. 14). Vor diesem Hintergrund scheint nachvollziehbar, dass ,Politik“ als Schlagwort eher selten ganz an den Anfang gestellt wurde, während gleichzeitig eine gründlichere Lektüre offenbart, dass Perspektiven auf Sozialpolitik (wenn auch teilweise implizit) immer irgendwie involviert waren.

Dies erscheint folgerichtig, wenn man davon ausgeht, dass Konstruktionen sozialer Probleme kaum unabhängig von ihrem politisch-ökonomischen Kontext verstanden werden können, dessen entscheidender Bestandteil in Gegenwartsgesellschaften staatlich organisierte Sozialpolitik ist. Im Übrigen weist Sozialpolitik - wie oben näher ausgeführt - als spezifische „Institution der Normativität“ (Groenemeyer 2008) diverse Bezugspunkte zu anderen Logiken der gesellschaftlichen Problemzuschreibung und -bearbeitung auf, so v. a. in professionellen Kontexten von Sozialer Arbeit und Gesundheitsberufen, aber auch an der Schnittstelle zu Institutionen von Kriminalität und Strafe oder einem präventiven Risikomanagement. Unter den Bedingungen eines „liberalisierten Wohlfahrtskapitalismus“ (Betzelt und Bode 2017) haben letztere Verbindungslinien in den zurückliegenden Jahrzehnten noch einmal deut- 
lich an Relevanz gewonnen. Zudem wurde erläutert, dass Sozialpolitik - schon auf der Ebene der zugrundeliegenden Wertvorstellungen und Kulturideen - von zahlreichen Widersprüchen geprägt und zudem in ständigem Wandel begriffen ist (vgl. Pfau-Effinger 2019). Schon auf der Makroebene zeigt sich also ein hohes Maß an institutioneller Komplexität und Dynamik.

Im Weiteren wurde dafür sensibilisiert, dass sich Sozialpolitik nicht nur als unmittelbare Arbeit an Institutionen - etwa im Rahmen von „claimsmaking“, ,entrepreneurship“ oder in der Auseinandersetzung von Parteien und Verbänden - vollzieht, sondern entscheidend durch die Arbeit an Menschen geprägt wird, die alltäglich vor Ort in Sozialverwaltungen und sozialen Dienstleistungsorganisationen stattfindet. Wie die o.g. Beispiele verdeutlichen, prallen auch hier - nicht zuletzt unter dem Einfluss einer veränderten organisationalen Infrastruktur im Sozial- und Gesundheitssektor (Bode 2013) - regelmäßig unterschiedliche, teilweise widersprüchliche Logiken aufeinander. Nicht nur die Makroebene politischer Auseinandersetzung und Programmentwicklung, sondern auch die Mikroebene sozialer Dienstleistungserbringung ist also notorisch von Komplexität gekennzeichnet. Für die soziologische Forschung und Theoriebildung scheint es generell ertragreich, diese Komplexität auf beiden Ebenen zur Kenntnis zu nehmen und idealerweise im Verbund zu betrachten (vgl. Friedland und Alford 1991, S. $240 \mathrm{ff}$.). Daraus erwachsen beidseitige Anschlussstellen für die Soziologie sozialer Probleme und die Soziologie der Sozialpolitik. Theoretisch-konzeptionell wurde im vorliegenden Beitrag unter Bezug auf neuere Strömungen des soziologischen Neo-Institutionalismus auf mögliche Ansätze verwiesen, die vielleicht auch dazu beitragen könnten, ,objektivistische " und ,konstruktivistische“ Perspektiven in der Soziologie sozialer Probleme ein Stück weit zu ,versöhnen', indem sie Institutionen in ihrer Wirkmächtigkeit, gleichzeitig aber auch in ihrer Konstruiertheit und prinzipiellen Veränderlichkeit in den Blick nehmen.

Methodisch spricht vieles für eine Kombination von Instrumenten, die wohlfahrtsstaatliche Institutionen und Diskurse (sowie deren Wandel) auf der Makroebene berücksichtigen, mit solchen, die in der Lage sind, die ,black box ' der Arbeit an sozialen Problemen in den Organisationen vor Ort zu erhellen. Als Beispiel für einen geeigneten Weg, der beide Ebenen unmittelbar integriert, sei hier nur der Ansatz der „institutionellen Ethnografie“ genannt, der bei der alltagweltlichen Praxis ansetzt und (oft anhand von Dokumenten) gleichzeitig die organisationalen und institutionellen Bedingungen dieser Praxis fokussiert (vgl. grundlegend Smith 2005; Nadai 2012; sowie für ein aktuelles Beispiel aus dem deutschsprachigen Raum Kühne und Schlepper 2020). Ein solches Forschungsprogramm bietet die Chance, einen Mainstream zu überwinden, der immer noch dazu neigt, ,zwischen Nahaufnahme und Totale zu oszillieren“ (Nadai 2012, S. 149) und dabei - in der einen oder anderen Weise - letztlich oft ein unvollständiges Bild vermittelt.

Zudem liegt darin nicht zuletzt ein kritisches Potenzial, das zwar ,die ,Konstruktionsmaschinerie‘ sozialer Praxis nicht zum Stillstand bringen, aber immerhin ihre Mechanismen freilegen und damit denen, die in ihrem Räderwerk stecken, Denkwerkzeuge in die Hand geben [kann]“ (dies., S. 162). Auch der mikrofundierte Neo-Institutionalismus sensibilisiert dafür, dass diejenigen, die Sozialpolitik vor Ort konkret ins Werk setzen, weder als „,cultural dopes“, die lediglich blind Routinen folgen, noch als ,heroische“ Individuen, die aus eigener Kraft die Welt verändern 
können, angemessen zu fassen sind (Powell und Colyvas 2008, S. 277). Vielmehr dürfte die Wahrheit irgendwo dazwischen liegen. Nimmt man Lipskys Diktum, dass Sozialpolitik in letzter Konsequenz auf ,street level“ gemacht wird, wörtlich, ist die alltägliche Arbeit in sozialen Dienstleistungsorganisationen und staatlichen Kontrollagenturen jedenfalls - in der einen oder der anderen Richtung - als politisches Handeln zu begreifen. So ist z.B. einerseits denkbar, dass Benachteiligungen und Dynamiken des sozialen Ausschlusses an der Basis (re)produziert werden, andererseits bieten sich aber Chancen für Widerstand gegen sozialpolitische Programme, die dies vorantreiben. Die Soziologie kann Fachkräften vor Ort Reflexionsangebote zur Verfügung stellen. Um das Beste aus der Situation zu machen, brauchen diese aber auch die Unterstützung der, großen Politik‘.

Funding Open Access funding enabled and organized by Projekt DEAL.

Open Access Dieser Artikel wird unter der Creative Commons Namensnennung 4.0 International Lizenz veröffentlicht, welche die Nutzung, Vervielfältigung, Bearbeitung, Verbreitung und Wiedergabe in jeglichem Medium und Format erlaubt, sofern Sie den/die ursprünglichen Autor(en) und die Quelle ordnungsgemäß nennen, einen Link zur Creative Commons Lizenz beifügen und angeben, ob Änderungen vorgenommen wurden.

Die in diesem Artikel enthaltenen Bilder und sonstiges Drittmaterial unterliegen ebenfalls der genannten Creative Commons Lizenz, sofern sich aus der Abbildungslegende nichts anderes ergibt. Sofern das betreffende Material nicht unter der genannten Creative Commons Lizenz steht und die betreffende Handlung nicht nach gesetzlichen Vorschriften erlaubt ist, ist für die oben aufgeführten Weiterverwendungen des Materials die Einwilligung des jeweiligen Rechteinhabers einzuholen.

Weitere Details zur Lizenz entnehmen Sie bitte der Lizenzinformation auf http://creativecommons.org/ licenses/by/4.0/deed.de.

\section{Literatur}

Albrecht, Günter. 1990. Theorie sozialer Probleme im Widerstreit zwischen „objektivistischen“ und ,rekonstruktionistischen“ Ansätzen. Soziale Probleme 1:5-20.

Altreiter, Carina, und Bettina Leibetseder. 2015. Constructing inequality: Deserving and undeserving clients in Austrian social assistance offices. Journal of Social Policy 44:127-145.

Anttonen, Anneli, und Jorma Sipilä. 1996. European social care services: is it possible to identify models? Journal of European social policy 6:87-100.

Bäcker, Gerhard, Gerhard Naegele, Reinhard Bispinck, Klaus Hofemann, und Jennifer Neubauer. 2010. Sozialpolitik und soziale Lage in Deutschland, 5. Aufl., Bd. 1. Wiesbaden: Springer VS.

Berger, Peter L., und Thomas Luckmann. 1969. Die gesellschaftliche Konstruktion von Wirklichkeit. Eine Theorie der Wissenssoziologie. Frankfurt a.M.: Fischer.

Betzelt, Sigrid, und Ingo Bode. 2017. Fatal funktional? Angstmobilisierung im liberalisierten Wohlfahrtskapitalismus. Leviathan 45:192-220.

Bode, Ingo. 2013. Die Infrastruktur des postindustriellen Wohlfahrtsstaats: Organisation, Wandel, gesellschaftliche Hintergründe. Wiesbaden: Springer VS.

Bode, Ingo, und Hannu Turba. 2014. Organisierter Kinderschutz in Deutschland. Strukturdynamiken und Modernisierungsparadoxien. Wiesbaden: Springer VS.

Bode, Ingo, und Hannu Turba. 2020. Schizophrenic sensemaking as (non-) response to institutional ambiguity: the case of the German child protection industry. Human Service Organizations: Management, Leadership \& Governance 44:9-31.

Böhringer, Daniela, Ute Karl, Hermann Müller, Wolfgang Schröer, und Stephan Wolff. 2012. Den Fall bearbeitbar halten: Gespräche in Jobcentern mit jungen Menschen. Opladen: Barbara Budrich. 
Dahme, Heinz-Jürgen, und Norbert Wohlfahrt. 2011. Zwang und Strafe als Mittel der Sozialpolitik. In Gerechte Ausgrenzung? Wohlfahrtsproduktion und die neue Lust am Strafen, Hrsg. Bernd Dollinger, Henning Schmidt-Semisch, 207-226. Wiesbaden: Springer VS.

DiMaggio, Paul. 1988. Interest and agency in institutional theory. In Institutional patterns and organizations: Culture and environment, Hrsg. Lynne Zucker, 3-22. Cambridge: Ballinger.

Dollinger, Bernd. 2007. Sozialpolitik als Instrument der Lebenslaufkonstitution - Argumente für eine Perspektivenveränderung. Zeitschrift für Sozialreform 53:147-164.

Drepper, Thomas. 2010. Soziale personenbezogene Dienstleistungsorganisationen aus neoinstitutionalistischer Perspektive. In Soziale personenbezogene Dienstleistungsorganisationen, Hrsg. Thomas Klatetzki, 129-164. Wiesbaden: Springer VS.

Duprez, Dominique, und Axel Groenemeyer, Hrsg. 2009. Drogenkonsum, Drogenprobleme und Drogenpolitik in Europa. Soziale Probleme 20 (1/2).

Floerecke, Peter, und Jürgen Mansel. 1990. Die Medien als Organ demokratischer Kontrolle staatlicher Politik am Beispiel der Gesetzgebungsverfahren zum Demonstrationsstrafrecht. Soziale Probleme $1: 36-61$

Friedland, Roger, und Robert Alford. 1991. Bringing Society Back In: Symbols, Practices, and Institutional Contradictions. In The new institutionalism in organizational analysis, Hrsg. Walter Powell, Paul DiMaggio, 232-266. Chicago: University of Chicago Press.

Garland, David. 2001. The culture of control: crime and social order in contemporary society. Chicago: University of Chicago Press.

Gilbert, Neil, Nigel Parton, und Marit Skivenes. 2011. Changing patterns of Response and Emerging Orientations. In Child protection systems. International trends and orientations, 243-257. New York: Oxford University Press.

Greenwood, Royston, Mia Raynard, Fara Kodeih, Evelyn Micelotta, und Michael Lounsbury. 2011. Institutional Complexity and Organizational Responses. The Academy of Management Annals 5:317-371.

Groenemeyer, Axel. 2001. Von der Sünde zum Risiko? - Bilder abweichenden Verhaltens und die Politik sozialer Probleme am Ende des Rehabilitationsideals. Soziale Probleme 12:146-182.

Groenemeyer, Axel. 2006. Gesellschaftspolitische Relevanz und soziologische Reputation. Eine kleine Geschichte über 30 Jahre Soziologie sozialer Probleme in Deutschland. Soziale Probleme 17:9-19.

Groenemeyer, Axel. 2008. Institutionen der Normativität. In Soziologie sozialer Probleme und sozialer Kontrolle. Realitäten, Repräsentationen und Politik, Hrsg. Axel Groenemeyer, Silvia Wieseler, 70-97. Wiesbaden: Springer VS.

Groenemeyer, Axel. 2010. Doing Social Problems - Doing Social Control. Mikroanalysen der Konstruktion sozialer Probleme in institutionellen Kontexten - Ein Forschungsprogramm. In Doing Social Problems, Hrsg. Axel Groenemeyer, 13-56. Wiesbaden: Springer VS.

Groenemeyer, Axel, Christoph Hohage, und Melanie Ratzka. 2012. Die Politik sozialer Probleme. In Handbuch soziale Probleme, 2. Aufl., Hrsg. Günter Albrecht, Axel Groenemeyer, 117-191. Wiesbaden: Springer VS.

Gurr, Thomas. 2018. Vergeltung, Ahndung, Integrationsversprechen: Sanktionen im SGB II. In Angst im neuen Wohlfahrtsstaat, Hrsg. Sigrid Betzelt, Ingo Bode, 249-274. Baden-Baden: Nomos.

Hasenfeld, Yeheskel. 2000. Organizational forms as moral practices: the case of welfare departments. Social Service Review 74:329-351.

Hasenfeld, Yeheskel. 2010a. The attributes of human service organizations. In Human services as complex organisations, Hrsg. Yeheskel Hasenfeld, 9-32. Thousand Oaks: SAGE.

Hasenfeld, Yeheskel. 2010b. Worker-client relations. Social policy in practice. In Human services as complex Organisations, Hrsg. Yeheskel Hasenfeld, 405-425. Thousand Oaks: SAGE.

Hasse, Raimund, und Lucia Schmidt. 2010. Unternehmertum, Arbeit, Sprache. Zur Mikrofundierung des Neo-Institutionalismus. Sociologia Internationalis 48:1-28.

Hilgartner, Stephen, und Charles Bosk. 1988. The rise and fall of social problems: a public arenas model. American Journal of Sociology 94:53-78.

Karstedt, Susanne. 1997. Recht und soziales Kapital im Wohlfahrtsstaat. Soziale Probleme 8:103-137.

Kaufmann, Franz-Xaver. 1973. Sicherheit als soziologisches und sozialpolitisches Problem. Stuttgart: Enke.

Kaufmann, Franz-Xaver. 2002. Elemente einer soziologischen Theorie sozialpolitischer Intervention. In Sozialpolitik und Sozialstaat. Soziologische Analysen, Hrsg. Franz-Xaver Kaufmann, 69-106. Wiesbaden: Springer VS.

Kaufmann, Franz-Xaver. 2012. Konzept und Formen sozialer Intervention. In Handbuch soziale Probleme, 2. Aufl., Hrsg. Günter Albrecht, Axel Groenemeyer, 1285-1305. Wiesbaden: Springer VS.

Klimke, Daniela. 2013. Die Politische Ökonomie der Sicherheit. Soziale Probleme 24:137-163. 
Krüger, Jürgen. 1994. Sozialstrukturelle Modernisierung: Stabilisierung oder Destruierung des Wohlfahrtsstaates. Soziale Probleme 5:5-30.

Kühne, Sylvia, und Christina Schlepper. 2020. „Meine Aufgabe ist, einen Bericht zu schreiben“: „Die sanften Kontrolleure" und ihre Dokumente. Soziale Probleme 30:145-168.

Lampe, Dirk. 2016. Ein goldenes Zeitalter des Jugendstrafrechts? Politische Debatten über Jugendkriminalität in den 1970er und 1980er Jahren in der Bundesrepublik. Soziale Probleme 27:95-118.

Lawrence, Thomas, und Roy Suddaby. 2006. Institutions and institutional work. In The sage handbook of organization studies, Hrsg. Stewart Clegg, Cynthia Hardy, und Thomas Lawrence, 215-254. London: SAGE.

Lessenich, Stephan. 2019. Sozialpolitik als Problemlöser und Problemverursacher. In Handbuch Sozialpolitik, Hrsg. Herbert Obinger, Manfred Schmidt, 883-901. Wiesbaden: Springer VS.

Lipsky, Michael. 2010. Street-level bureaucracy. Dilemmas of the individual in public services. 30th anniversary expanded edition. New York: SAGE.

Ludwig-Mayerhofer, Wolfgang, Olaf Behrend, und Ariadne Sondermann. 2009. Auf der Suche nach der verlorenen Arbeit: Arbeitslose und Arbeitsvermittler im neuen Arbeitsmarktregime. Konstanz: UVK.

Mierendorff, Johanna, und Ilona Ostner. 2014. Kinder im Wohlfahrtsstaat. Leitbilder der aktuellen Sozialpolitik. In Kinderschutz. Wie kindzentriert sind Programme, Praktiken, Perspektiven?, Hrsg. Doris Bühler-Niederberger, Lars Alberth, und Steffen Eisentraut, 200-221. Weinheim, Basel: Beltz Juventa.

Miller, Gale, und James Holstein (Hrsg.). 1997. Social problems in everyday life. Studies in social problems work. Greenwich: JAI Press.

Nadai, Eva. 2012. Von Fällen und Formularen: Ethnographie von Sozialarbeitspraxis im institutionellen Kontext. In Kritisches Forschen in der Sozialen Arbeit, Hrsg. Elke Schimpf, Johannes Stehr, 149-163. Wiesbaden: Springer VS.

Nothdurfter, Urban, und Koen Hermans. 2018. Meeting (or not) at the street level? A literature review on streetlevel research in public management, social policy and social work. International Journal of Social Welfare 27:294-304.

Nullmeier, Frank. 2019. Begründungen des Wohlfahrtsstaates. In Handbuch Sozialpolitik, Hrsg. Herbert Obinger, Manfred Schmidt, 57-75. Wiesbaden: Springer VS.

Peters, Helge, und Helga Cremer-Schäfer. 1975. Die sanften Kontrolleure. Wie Sozialarbeiter mit Devianten umgehen. Stuttgart: Enke.

Pfau-Effinger, Birgit. 2019. Kulturelle Ideen als Grundlage der Wohlfahrtsstaatsforschung. In Handbuch Sozialpolitik, Hrsg. Herbert Obinger, Manfred Schmidt, 217-234. Wiesbaden: Springer VS.

Powell, Walter, und Jeannette Colyvas. 2008. Microfoundations of institutional theory. In The sage handbook of organizational institutionalism, Hrsg. Royston Greenwood, Christine Oliver, Roy Suddaby, und Kerstin Sahlin, 276-298. Thousand Oaks: SAGE.

Ratzka, Melanie. 2005. Politik gegen sozialräumlich konzentrierte Benachteiligung - Ein Fallbeispiel. Soziale Probleme 16:54-73.

Sack, Fritz. 2006. Gesellschaftliche Entwicklung und Sanktionseinstellungen - Anmerkungen zur deutschen kriminalpolitischen Diskussion. Soziale Probleme 17:155-173.

Schäfers, Markus. 2014. „Personenzentrierung“ als sozialpolitische Programmformel im Zeichen der Inklusion. Zu den Widersprüchlichkeiten einer Neuausrichtung des Hilfesystems für Menschen mit Behinderungen. Soziale Probleme 25:317-338.

Schmidt, Lucia, und Raimund Hasse. 2010. Der Arbeitsbegriff in der Soziologie sozialer Probleme und im Neo-Institutionalismus. Konzeptualisierung und Anwendung im Forschungsfeld Bildungsungleichheit. In Doing Social Problems, Hrsg. Axel Groenemeyer, 57-78. Wiesbaden: Springer VS.

Skivenes, Marit. 2011. Norway: toward a child-centric perspective. In Child protection systems: international trends and orientations, Hrsg. Neil Gilbert, Nigel Parton, und Marit Skivenes, 154-180. New York: Oxford University Press.

Smith, Dorothy. 2005. Institutional ethnography: a sociology for people. Lanham: Rowman Altamira.

Spector, Malcolm, und John Kitsuse. 1977. Constructing social problems. New York: de Gruyter.

Sucherdt, Christoph. 2020. Zur soziologischen Auseinandersetzung mit Gewalt in der Medizin am Beispiel des medizinischen Kinderschutzes. Österreichische Zeitschrift für Soziologie 45:69-88.

Thornton, Patricia, William Ocasio, und Michael Lounsbury. 2012. The institutional logics perspective. A new approach to culture, structure and process. Oxford: Oxford University Press.

Turba, Hannu. 2018. Die Polizei im Kinderschutz: Zur Verarbeitung institutioneller Komplexität in hybriden Berufswelten. Wiesbaden: Springer VS.

Turba, Hannu, Janne Breimo, und Christian Lo. 2019. Professional and organizational power intertwined: barriers to networking? Children and Youth Services Review 107:1-9. 
Wacquant, Loïc. 2013. Bestrafen der Armen: zur neoliberalen Regierung der sozialen Unsicherheit. Opladen: Barbara Budrich.

Wiedemeyer, Michael, Kai Hauprich, und Thomas Münch. 2018. Angst im Jobcenter? Aktuelle Befunde zur Alltagspraxis der Aktivierung. In Angst im neuen Wohlfahrtsstaat, Hrsg. Sigrid Betzelt, Ingo Bode, 319-338. Baden-Baden: Nomos.

Wolff, Reinhart. 2017. Moderner Kinderschutz - ganzheitliche Hilfe oder autoritäres Risikomanagement? In Modernes Leben - Leben in der Moderne, Hrsg. Stefan Busse, Kornelia Beer, 181-199. Wiesbaden: Springer VS.

Zahradnik, Franz. 2018. Schamangst und Sanktionierung. Affektive Verstrickungen junger Arbeitsloser in die Widersprüche der Grundsicherung. In Angst im neuen Wohlfahrtsstaat, Hrsg. Sigrid Betzelt, Ingo Bode, 275-294. Baden-Baden: Nomos.

Zahradnik, Franz, Franziska Schreyer, Andreas Moczall, Lutz Gschwind, und Mark Trappmann. 2016. Wenig gebildet, viel sanktioniert? Zur Selektivität von Sanktionen in der Grundsicherung des SGB II. Zeitschrift für Sozialreform 62:141-180. 